FERMILAB-TM-2014

\title{
Switchyard in the Main Injector Era Conceptual Design Report
}

\author{
C. Brown, T. Kobilarcik, P. Lucas, A. Malensek, C.T. Murphy and M.-J. Yang
}

Fermi National Accelerator Laboratory

P.O. Box 500, Batavia, Illinois 60510

August 1997 


\section{Disclaimer}

This report was prepared as an account of work sponsored by an agency of the United States Government. Neither the United States Government nor any agency thereof, nor any of their employees, makes any warranty, express or implied, or assumes any legal liability or responsibility for the accuracy, completeness or usefulness of any information, apparatus, product or process disclosed, or represents that its use would not infringe privately owned rights. Reference herein to any specific commercial product, process or service by trade name, trademark, manufacturer or otherwise, does not necessarily constitute or imply its endorsement, recommendation or favoring by the United States Government or any agency thereof. The views and opinions of authors expressed herein do not necessarily state or reflect those of the United States Government or any agency thereof.

\section{Distribution}

Approved for public release: further dissemination unlimited. 


\title{
Switchyard in the Main Injector Era Conceptual Design Report
}

\author{
C. Brown, T. Kobilarcik, P. Lucas, \\ A. Malensek, C.T. Murphy and M.-J. Yang
}

\section{Introduction}

This report presents elements of a design of the Switchyard and of the present fixed target beamlines in the era of the Main Injector (MI). It presumes that $800 \mathrm{GeV}$ Tevatron beam will be transported to this area in the MI era, and permits it to share cycles with $120 \mathrm{GeV}$ Main Injector beam if this option is desired. Geographically, the region discussed extends from the vicinity of $A 0$ to downstream points beyond which beam properties will be determined by the requirements of specific experiments. New neutrino lines not utilizing the present Switchyard (NuMI, BooNE) are not addressed. Similarly Main Injector beams upstream of $\mathrm{AO}$ are described fully in $\mathrm{MI}$ documentation and are unaffected by what is presented here.

The timing both of the preparation of this report and of its recommendations for proceeding with construction relate to a desire to do required work in Transfer Hall and Enclosure B during the Main Injector construction shutdown (September 1997 - September 1998). As these areas are off-limits during any Tevatron operation, it is necessary for the fixed target program that work be completed here during this extended down period.

The design presented here enables the operation of all beamlines in the manner specified in the current Laboratory plans for future fixed-target physics. The current plans are specified in Table I.

Table I

Anticipated utilizations of fixed-target beams

\begin{tabular}{|l|l|}
\hline Proton & $800 \mathrm{GeV}$ \\
\cline { 2 - 2 } & No $120 \mathrm{GeV}$ \\
\cline { 2 - 2 } & Not necessarily 3-way split \\
\hline Muon (NM2) & $120 \mathrm{GeV}$ and $800 \mathrm{GeV}$ with a changeover period \\
\hline Neutrino & Program moves to new areas being designed \\
\cline { 2 - 2 } & Recycle components \\
\hline Meson & No $800 \mathrm{GeV}$ \\
\cline { 2 - 2 } & $120 \mathrm{GeV}$ \\
\cline { 2 - 2 } & Maintain 3-way split \\
\end{tabular}

The period 1998-1999

The assumption for this time frame is that the Main Injector will be feeding the Tevatron for the continuation of the $800 \mathrm{GeV}$ fixed target run in KTeV and perhaps the Proton area. Therefore, $800 \mathrm{GeV}$ transport and splitting between Proton and NM2 (the targeting enclosure for $\mathrm{KTeV} / \mathrm{KAMI}$ ) are maintained. However, even with this task plus perhaps that of antiproton stacking, the new machine will have the capacity to accelerate still more protons. We intend to use this capability to commission MI slow extraction and beam 
transfer to the Switchyard; thus there will be both Tevatron and MI beam in the Switchyard over this period. One interesting possibility is to send MI beam during the 40 seconds of each cycle for which the Tevatron is ramping down and back up. If this goal turns out to be impractical, then we intend to use study time when the Tevatron is off for MI fixed target commissioning. It is not envisioned that during this period MI beam will be used for other than commissioning or have any destination other than the Switchyard dump. However, we feel that it is very important to measure the properties of the $120 \mathrm{GeV}$ beam as it enters Switchyard in order to make realistic - and economical - final choices for the quadrupole optics necessary to keep it adequately small in the Meson and NM2 lines.

The period 2000 and beyond

The general assumption for the longer term program is that it will consist firstly of $120 \mathrm{GeV}$ beam to Meson. This beam will be for the CPT and CKM experiments, for a possible NUMI particle production measurement and for one or more test beams. Secondly, it will consist of $120 \mathrm{GeV}$ beam to NM2 (for KAMI). Lastly, it is presumed that the $800 \mathrm{GeV}$ fixed target program will be completed. However, the capability of sending $800 \mathrm{GeV}$ beam to the Proton area and to NM2 is maintained, in the case that $800 \mathrm{GeV}$ experiments in either area are approved during some future collider upgrade period. The PLAMs could be removed to avoid further irradiating them and replaced only when and if $800 \mathrm{GeV}$ beam goes to Proton again.

This and many present capabilities can be retained and others added at essentially no cost. For example, if it were desired to direct $120 \mathrm{GeV}$ beam to Proton, this could be accomplished by moving two of the PSEPs from the present Tevatron/Switchyard line to the new A0 $120 \mathrm{GeV}$ bypass (see below). If it is desired still to transport protons to the test beam in NW, this is possible simply by not removing the MUSEP split. However in both of these cases the total beam to Switchyard would have to be low intensity (of order $3 \mathrm{x}$ $10^{12}$ ), as the beam does not clear the PSEPs and MUSEPs in the optics presented here so well as it clears the MSEPs and FSEPs. We emphasize that neither of these two options is being planned.

The assumptions for the Meson Lab are that beam requests there will originally be of order $1 \times 10^{12}$ per spill and that they will escalate over time to near $5 \times 10^{12}$; it is presumed that at any one time there will be two or more Meson experiments active. The further presumption is that the cryogenic left bends will be removed prior to this time period (see below), and that the new beamline described in this report will be utilized. The KAMI beam request is expected to be a few $\times 10^{13}$, but beyond this specification little can yet be said about the KAMI line.

Therefore, the shielding through the Switchyard to the KAMI target will be made adequate for the transport of $3 \times 10^{13}$ protons every 3 seconds. The shielding for the transport after the KAMI split to the Meson Lab will be certified for only $5 \times 10^{12}$ protons.

Figure 1 shows the beams that are planned for the year 2000 and beyond.

\section{Design of F48 through Enclosure B}

This section gives details of the design of the $120 \mathrm{GeV}$ A0 bypass beamline, as shown in Figure 2 (plan view) and Figure 3 (elevation view). The task in this design has been to get $120 \mathrm{GeV}$ beam out of the Main Ring remnant (F11-F48) and have it join the Switchyard at an appropriate point. The space between the end of the string of Proton line electrostatic 
septa (PSEPs) and the beginning of the VH94 string (see Figure 2) was chosen for two reasons. This is the only available drift space early in the Switchyard long enough to allow bringing in a second beam from a trajectory different from that of Tevatron extraction. Since there are no immediate plans to split $120 \mathrm{GeV}$ beam between Proton and MesonNeutrino, it is acceptable to reenter the Switchyard downstream of the PSEPs.

At least four schemes of how to reenter the Switchyard at this point have been studied. The constraints which we have imposed on all schemes are:

1. Do not set any new magnets at places which block access to the PSEPs or the Tevatron Lambertson magnets and skew dipoles;

2. Minimize the number of Switchyard magnets which need to be relocated;

3. Minimize the number of new magnets added;

4 Change from $800 \mathrm{GeV}$ Tevatron extraction to $120 \mathrm{GeV}$ MI extraction without an access to the tunnel.

What economies could be achieved by removing the last constraint are examined at the end of this section.

Of the schemes studied, the bypass beamline presented here best satisfies these constraints. In fact only one of the Switchyard magnets through Enclosure B needs to be replaced. We now describe the layout (Figures 2 and 3) in detail.

\section{Main Ring modifications}

The F48-4 and -5 dipoles are removed so that the beam will angle westward through the Transfer Hall. The remaining F48-3 dipole is rolled by $7^{0}$ to give the beam a small necessary down bend (see Figure 3). The existing quadrupole doublet at F49 is moved west by an amount ranging from 4 to 10 inches. This doublet remains an important element in focusing the extracted beam; it will have its polarity reversed to agree with the Tevatron (see optics discussion below) and may have the separation of the two halves changed to optimize the optics into the rest of Switchyard. The existing MR trim magnets at F49 have adequate trim power to correct for the geometry and alignment uncertainties in this region.

\section{Bypass line magnets}

Next to Switchyard magnet V91, an EPB dipole steers the beam back toward the gap between the PSEPs and VH94. In that same region there is also added an EPB quadrupole to play the same role as $\mathrm{Q} 90$ in the $800 \mathrm{GeV}$ extraction line, and a vertical trim dipole to correct for misalignment of the quadrupole and to center the beam at VH94. A SWIC is needed just upstream of these three magnets. If further optics studies indicate the need for another quadrupole here (to change from a singlet to a doublet), there is room for one at the upstream end of this area.

The new magnets mentioned in the above paragraph will need new power supplies and cabling from these power supplies to the magnets. Although we call the magnets and power supplies "new", the word "recycled" is more appropriate. We believe that we can obtain all of them from a combination of the current not-installed inventory and magnets and power supplies removed from beamlines not scheduled to run beyond the fall of 1997 .

Despite the addition of these new elements in what is currently the aisle, there is still $73^{\prime \prime}$ of clearance to the west wall. The new beam line clears by a large amount the power feed can (not shown in Figure 2) which sits in the aisle opposite V90. The beam pipe has a slight overlap with parts of the PSEP vacuum pump just downstream of V91, but we envisage several easy ways to reconfigure these vacuum parts. The beam clears the end of the last PSEP vacuum tank by $2.1^{\prime \prime}$, which still allows the usual 4 " beam pipe. 


\section{Rejoining Switchyard}

Just upstream of VH94, two EPB dipoles are installed and direct the $120 \mathrm{GeV}$ extracted beam back onto the Switchyard trajectory. They are rolled $14^{0}$ to give the beam a slight up bend. They will need a special $4^{\prime \prime}$ wide vacuum can in order to admit both beams comfortably (see Figure 2). The $800 \mathrm{GeV}$ beam, which does not use these magnets, is offset further from the magnetic centerline than the $120 \mathrm{GeV}$ beam in order that the 120 $\mathrm{GeV}$ beam avoid the bad field region of the EPB dipoles. Figure 4 shows what these magnets look like in the tunnel. They give adequate personnel passage and do not interfere with the cable trays. Just upstream of the EPBs a 4-4-30 vertical trim magnet allows vertical fine tuning; horizontal fine tuning is achieved in the EPB magnet power supplies, with a small amount of vertical coupling which is removed by the vertical trim.

A few obsolete beam elements need to be removed from this region to accommodate the $120 \mathrm{GeV}$ beam. PV92 (needed only for fast spill) and VT92 (unused) will be removed. If a fast spill experiment is approved during the 1998-1999 $800 \mathrm{GeV}$ fixed target run, a $1.5^{\prime \prime}$ $x$ 40" trim magnet (namely the present VT92) will fit at the location of PV92 and is powerful enough for the required fast spill bump. Two redundant beam position monitors just upstream of VH94 will be removed. The SWIC at VH94 remains, occupying the existing 18 "long box.

Table II shows the new or altered magnets in the bypass line and their bend angles and currents. The totals are 6 new magnets and 4 new power supplies. The total DC power for the magnets labeled "new" is $65 \mathrm{~kW}$, and we estimate that we can reduce that by a factor two with fast ramps, even on a $3 \mathrm{sec}$ cycle. A round number cost estimate for installation of the A0 bypass beamline is $\$ 180 \mathrm{~K}$, apportioned $44 \%$ parts and $56 \%$ labor.

Table II

Magnets involved in the bypass beamline with their bend angles and currents

\begin{tabular}{|lccccl|}
\hline \multicolumn{1}{|c}{ Type } & $\begin{array}{c}\text { Location } \\
\text { feet }\end{array}$ & $\begin{array}{c}\text { Bend } \\
\text { mrad }\end{array}$ & $\begin{array}{c}\text { Current } \\
\text { amp }\end{array}$ & $\begin{array}{c}\text { Max. bend } \\
\text { mrad }\end{array}$ & Comment \\
\hline \hline $\begin{array}{l}\text { MR dipole F48-3 } \\
\text { (East bend) }\end{array}$ & -163.98 & 8.118 & 1360.0 & & $\begin{array}{l}\text { existing on MR bus, } \\
\text { rolled 7.416 }\end{array}$ \\
\hline MR horiz trim at F49 & $\sim-98.5$ & 0.000 & 0.0 & 0.103 & existing \\
\hline MR vert trim at F49 & $\sim-98.5$ & 0.000 & 0.0 & 0.038 & $\begin{array}{l}\text { replace existing with } \\
\text { normal strength vert }\end{array}$ \\
\hline $\begin{array}{l}\text { 2-5-40 vert trim next to } \\
\text { H90 }\end{array}$ & 133.30 & 0.000 & 0.0 & 0.630 & new magnet, new PS \\
\hline EPB quad next to Q90 & 140.97 & & 8.8 & & new magnet, new PS \\
\hline $\begin{array}{l}\text { EPB dipole next to V91 } \\
\text { (East bend) }\end{array}$ & 151.97 & 8.493 & 1064.0 & & new magnet, new PS \\
\hline 4-4-30 vert trim & 285.29 & 0.000 & 0.0 & 1.000 & new magnet, new PS \\
\hline $\begin{array}{l}\text { 2 EPB dipoles u.s. of VH94 } \\
\text { (West and Up) }\end{array}$ & 298.04 & 16.259 & 1018.0 & & $\begin{array}{l}\text { new magnets, in series } \\
\text { with dipole at V91, rolled } \\
14.442^{0}\end{array}$ \\
\hline
\end{tabular}

* This is the DUSAF Y coordinate of the center of the magnet. Our definition of this coordinate is that it equals zero at the Main Ring A0 and is angled westward from the original Main Ring beam at A0 by exactly $21.987 \mathrm{mrad}$. 
Simplification if there were no $800 \mathrm{GeV}$ extraction program

It was mentioned above that this scheme specifically allows switching between $120 \mathrm{GeV}$ and $800 \mathrm{GeV}$ extraction without a tunnel access. We have examined whether economies could be achieved by dropping this constraint, i.e., do not complete the installation of the A0 bypass line until the $800 \mathrm{GeV}$ program is complete and the PSEPs can be removed. In this case, the beam can be aimed more directly from the Main Ring towards the VH94 switch (see Figure 2b). In the Main Ring, dipoles F49-3 and -4 would be removed and a $10^{\prime}$ Main Ring dipole on the Main Ring bus would be added just upstream of F49-5, both rolled a few degrees to add a $1 \mathrm{mrad}$ down bend. The bypass horizontal dipole near V91 is not needed. Q90, V91, and H90-1 could be rigged from the $800 \mathrm{GeV}$ line into the $120 \mathrm{GeV}$ line to serve as the necessary bypass line quadrupole and trims, running off their existing power supplies. The VH94 string and its SWIC would have to be moved 52' upstream of their present locations, reduced to a two-magnet string, and rerolled to $6^{0}$ (presently $62^{\circ}$ ). In this scheme, only two new magnets are needed (the $10^{\prime} \mathrm{MR}$ dipole and the vertical trim at VH94) and one new power supply (for the vertical trim), compared to 6 new magnets and 4 new power supplies for the scheme of Figure 2 and Table II. The total power cost is reduced by $\$ 700 /$ month in ramped mode (assuming continuous $120 \mathrm{GeV}$ extraction every 3 seconds).

We do not regard this saving as significant compared to the total project (note the major rebuild of the Meson Line below). Furthermore, the opportunity to get an early look at beam spot sizes in 1999 - which could save the total project money by reducing the number of quadrupoles added - would be lost. Finally, to restore the ability to run $800 \mathrm{GeV}$ to Proton six magnets and a SWIC would have to be moved back into the $800 \mathrm{GeV}$ line several days of rigging and realignment.

\section{Components of the $120 \mathrm{GeV}$ Meson Line (Encl. $\mathrm{C}$ and beyond)}

The Meson branch of the Switchyard, through Enclosure C, the F1-3 manholes and the Meson area beamlines, was originally designed for operation at $200 \mathrm{GeV}$. As part of the Tevatron II upgrade in 1980 these beamlines were reconfigured for operation at $800 \mathrm{GeV}$. Nevertheless, the geometry of the Meson line and the Meson Detector Building is more appropriately sized for lower energies.

Designing an optimized $120 \mathrm{GeV}$ transport system through these enclosures involves reducing the length of most of the bend strings and adding more quadrupole focusing at strategic places. It also creates an opportunity to replace the cryogenic Doubler/Saver dipoles in the 10 degree west-bending 'left-bend' magnet string with conventional EPB Switchyard dipoles in order to save some cryogenic operating expenses (at the cost of additional electrical power).

\section{Replacement of the Meson cryogenic Left-Bend dipoles}

The $158 \mathrm{mr}$ left-bend is currently achieved by 21 Doubler/Saver 22-foot dipoles plus 4 EPB dipoles on a separate power supply (H201). In deciding how many EPB dipoles to use to replace the cryogenic magnets, we sought a solution which kept the current as low as possible, implying as many magnets as possible. On the other hand the number of magnets installed should be not so many as to cramp the space between the two halves of the bend. The purpose of this restriction was to allow room to install a $180^{\circ}$ phase advance FODO cell to keep the bend achromatic. The choice has been to install 24 new EPB dipoles, nine of which come from elsewhere in Enclosure $C$ where strings of four dipoles for $800 \mathrm{GeV}$ are reduced to one for $120 \mathrm{GeV}$, for a total of 28 dipoles in series. 
This solution requires a current of 709 amperes and 360 volts total (including bus-work). This current is low enough that we could use the existing 2-cable leads from the H201 power supply to get the power to the tunnel. One Transrex power supply on the 400 volt tap (two Transrexes if ramping) would provide the excitation. The total DC power (assuming $10 \%$ power supply loss) is $284 \mathrm{~kW}$, which would cost $\$ 11,000 /$ month. Carefully timed ramping with the 800 volts available from two Transrexes could cut this cost to $\$ 6,000 /$ month. Even if ramping is too difficult on the 3 second cycle (conversation with S. Orr), we would want it available to save power during any era with cycles "interleaved" between NUMI and Switchyard (see P. Martin and T. Murphy, Proceedings of the Main Injector Fixed Target Workshop, Fermilab, May 1997).

We can calculate the payback period if we assume a replacement of the 21 Doubler dipoles with 24 EPBs and no other changes. The costs to convert back to EPB dipoles are:

Mechanical (L. Sauer Memo) \$113,000

Electrical (J. Walton Memo) \$50,000

The total cost is about $\$ 200 \mathrm{~K}$ (after adding $25 \%$ contingency).

The cost/month to run the left bends is:

\begin{tabular}{lccc} 
& $\begin{array}{c}\text { Cryo-bends } \\
\text { Power }\end{array}$ & EPBs & \\
LN2 and LHe & $\$ 11,000$ & $\$ 6,000$ & $(\$ 11,000 \mathrm{DC})$ \\
Labor, supplies & $\$ 5,500$ & & \\
Total & $\$ 5,000$ & $-\$ 1,000$ & \\
\cline { 2 - 3 } & $\$ 21,500$ & $\$ 7,000$ & $(\$ 12,000 \mathrm{DC})$
\end{tabular}

Thus conventional EPB dipoles relieve between $\$ 14,500$ and $\$ 9,500$ in costs per month of running. The payback period is thus somewhere between one and two years of running.

Other features of the $120 \mathrm{GeV}$ Meson line

It will be more efficient and cost-effective if the replacement of the left-bend cryogenic dipoles is coordinated with other modifications in Enclosure $C$ needed to allow the transport of $120 \mathrm{GeV}$ beams through the Meson branch optics (see below). Some Lambertson magnets, vertical bend dipoles, trim dipoles, etc. are not needed at $120 \mathrm{GeV}$ beam energy and can be removed as part of a general Enclosure $\mathrm{C}$ project. There is also a need to clean up many years of 'barnacles' in the instrumentation and cabling runs in this enclosure.

It should be noted that none of the 21 cryogenic left-bend dipoles have ever failed, and that none of the six spare Doubler/Saver dipoles stored in Enclosure $C$ have been used after 15 years. Since manpower and funds for the changeover may be in short supply during FY1998, it makes sense to postpone the Enclosure C refit until later. Enclosure C does not need to be secured when the Tevatron is in collider mode, and hence this work could occur after the $800 \mathrm{GeV}$ fixed target run in 1999. This would also allow more time to identify, assemble, and test EPB dipoles, build the necessary hangers, decide the final quadrupole optics and identify the money needed for the full project.

\section{$120 \mathrm{GeV}$ Beam Optics}

In the Main Injector Technical Design Handbook the $120 \mathrm{GeV}$ slow spill beam line is described as consisting of four segments: MI-52 to F11, F11 to F17, F18 to F48 and F48 to Switchyard. The present work extends the design of this line, in Meson as far as the three-way Lambertsons and toward KTeV/KAMI to enclosure NM2. The optics have been 
studied over the line's entire length, albeit thus far with different procedures in different regions. The vicinity of F11 is one region which has been looked at especially carefully, and Figure 5 shows the proposed optics. The figure also indicates the repetitive Main Ring lattice which continues to the vicinity of F48.

In the new A0 bypass beamline described in detail above, the goal, from an optics point of view, is to provide agreement in $\alpha$ and $\beta$ with those parameters of the Tevatron. Those values for these parameters are required to minimize spot size at the critical aperture of the MSEPs. Figure 6 indicates the present state of this work, namely a demonstration of the extent to which both horizontal and vertical matches can be obtained by varying magnet currents in the region. Implicit in this figure is a polarity reversal of the MR F49 doublet. It is expected that a more complete solution will involve longitudinal magnet repositioning as well as current variation. This work is in progress.

Once into the Switchyard there are other considerations concerning satisfactory beam delivery. These include:

- Remove the cryogenic left bends, as has been discussed in detail above. The replacement frees space for new focusing elements or instrumentation as desired. As to optics, a goal is to maintain the achromaticity of the bend thus minimizing the effects of both beam energy spread and power supply variation due to ripple or misregulation.

- Maintain a vertical beam size at the MSEP and FSEP splitting stations small enough to minimize losses on the cathodes, noting that $\mathrm{MI} 120 \mathrm{GeV}$ beam is naturally larger in transverse size than that from the Tevatron at $800 \mathrm{GeV}$.

- Reposition the FSEP electrostatic septa which subdivide the Meson beam. Since $120 \mathrm{GeV}$ beam diverges more quickly in a drift space than $800 \mathrm{GeV}$ beam (because of the larger unnnormalized emittance), it is possible and necessary to move these septa downstream closer to the targets. This move will involve rigging work which must be included in evaluating the total project impact. However, space is gained into which focusing elements can be placed. After considerable discussion it has been decided to locate these septa in the M01 enclosure. This location will necessitate moving almost all beamline elements in Enclosures M01 and M02 as well as modifying the Meson target train.

Figure 7 shows the proposed Meson line optics from a position near A0 through enclosure M02; Figure 9 shows the corresponding information for the NM2 line. The envelope drawn for the vertical corresponds to a normalized emittance of $78 \pi \mathrm{mm}-\mathrm{mr}$ (corresponding to $99.7 \%$ of a $40 \pi$ beam), while that for the horizontal is for $40 \pi ; \delta p / p$ is $10^{-3}$. The difference between the two planes arises from the fact that with horizontal extraction from the Main Injector, the extracted beam at any point in time never samples the full horizontal emittance of that which is circulating. The $40 \pi$ value results from circumscribing a quite conservative ellipse around the extracted phase space. In Figures 8 and 10 are shown the same envelopes compared with the apertures of various devices encountered. A major challenge has been to achieve a fit through the MSEPs and FSEPs as well as the Meson Lambertsons.

Points of note:

- The currents in Q90, Q100 and Q101 at 500' have not been scaled linearly with momentum, but have been altered significantly to achieve the desired beam size through the MSEPs; in particular Q101 is not powered at all, but the second member of Q100 is put on a separate power supply to make a tightly spaced doublet. 
- The major left bend, effected by the two sets of magnets located at $1250^{\prime}$ and $1800^{\prime}$ in Meson, is made achromatic, as desired, by the insertion near its center of four quadrupoles leading to a $180^{\circ}$ phase advance.

- The tight fits in the vertical at 700' and 4000' for Meson are at the two sets of septa. The unusual appearance of the vertical apertures at these locations is due to careful inclusion of sagitta effects. It is concluded from this diagram, and given the conservative manner in which the beam envelope is drawn, that the aperture is of sufficient size. However there is almost no room for error indicated, and the need to take other action in these areas (mechanically changing the septa) has not been totally discounted. A high statistics ray tracing exercise is under way in order to better understand issues of tight apertures.

- There are quadrupole doublets in the downstream portions of the Meson line (physically located in the F-manholes) in a greater number than are present for Tevatron beam in the same region. The purpose is to maintain a tight focus to the FSEPs, which as noted have been moved downstream to Enclosure M01. The optics through these manholes will be quite similar to that which existed in the $200 \mathrm{GeV}$ era.

- Similarly, three quadrupole doublets have been added in the downstream portion of the line to NM2. The purpose of these is to keep the large $120 \mathrm{GeV}$ beam within all apertures. It is not possible to fit the assumed emittance through the apertures without such doublets being added; see Figure 11 for a typical plot.

- The KTeV/KAMI design presumes the removal of several pulsed devices. These are present for transport to Neutrino, a function which will no longer be required.

These optics for Meson require no new quadrupole magnets in Enclosure $\mathrm{C}$, only that the devices existing there be rearranged. However there is a requirement for five additional quadrupole power supplies. The F1-F3 manholes require five additional quadrupoles and power supplies. Attempts will continue to be made to minimize these numbers while maintaining the satisfactory focusing presented.

The optics for KAMI require the addition of three doublets in the NM line - one in Enclosure C, one in G2 and one in NM1. The Enclosure C doublet is formed by two 3 Q120s which must be rigged in. The G2 doublet is made of an existing 3Q120 (Q420) and another which must be rigged in. Because NM1 is a small enclosure which already contains a bend, its doublet is formed by two 3Q60s (which must be rigged in). Thus three additional 3Q120s and two additional 3Q60s will be used, together with three new power supplies. Cost and effort estimates for the reworking of beamlines in Enclosure $\mathrm{C}$ and beyond are in preparation.

\section{Shielding}

A shielding assessment is in the process of being performed assuming Main Injector beam through the entire region covered by this report, namely from F0 to the Meson Detector Building and KTev/KAMI hall. As compared with that of the Tevatron, MI beam requires more shielding. This is primarily due to the more rapid cycling of the $\mathrm{MI}$ as compared to the Tevatron, 2.9 seconds per slow spill cycle vs. 60 seconds, which has a greater effect than that of the lower beam energy, $120 \mathrm{GeV} v s .800 \mathrm{GeV}$. This assessment will be processed through all the proper channels of review and certification, with remediation added as appropriate. Although what has been done has not yet been subjected to this formal procedure, it is complete enough to indicate the magnitude of effort involved.

The precise criteria used for this assessment are $3 \times 10^{13}$ protons per pulse, with energy of $120 \mathrm{GeV}$ and cycle time of 2.9 seconds as stated above. In areas where both Tevatron and MI beams can be present, the Tevatron losses can add up to $15 \%$ to the MI dose rates. Note 
that the intensity to be sent to Meson is not expected to exceed $5 \times 10^{12}$ protons per pulse. Thus areas reachable by only Meson beam might be assessed at this lower level and protected against higher intensity accidents by interlocked detectors. However this is not the approach which has been taken for Meson in the past.

Three separate geographic regions are considered:

(a) F0 to Road D, where the shielding is part of the Main Injector construction project;

(b) Road D to the Master Substation, a region under the aegis of the former Accelerator, and now the Beams, Division;

(c) Downstream of the Substation to the Meson Detector building, formerly the territory of the Research Division but now the Beams Division. Similarly downstream of the MSEPs to the KTeV target hall.

Note that the final assessment must be done with magnets placed as indicated in this report (see subsections above) and that future magnet moves will have to be assessed properly.

Region (a) has progressed the farthest, having FESS drawings which show the entire longitudinal area, cross sections, and labyrinths. Also included are values which give the maximum overburden of earth cover which can be sustained from a structural point of view. Based on these drawings an assessment has been completed and solutions proposed to address the deficiencies found. The following is a summary of these results:

(1) No action is necessary at the four Refrigerator Buildings (F-1 to F-4) which are covered by existing "controlled entry procedures."

(2) No action is needed at the South Booster Road where it crosses over the ring; this area is already protected by interlocked detectors.

(3) Signs and fences are needed along the berm from F0 through the end of the Transfer Gallery. In addition interlocked detectors are required at a few places, including Transfer Gallery offices.

(4) One to three feet of soil must be added from the end of the Transfer Gallery to Road D.

(5) Either steel or interlocked detectors are required at Road D.

FESS drawings for region (b), comparable to those for region (a) utilized in the above, are in preparation with completion expected in late summer 1997. At that time region (b) will be assessed in a comparable manner to that above. There follow general statements of the expected results. First, the solutions for most of this region will be similar to those of region (a) - signs and fences, interlocked detectors and the addition of soil. Second, difficulties are likely to be found at the Road A/Road B intersection; all attempts will be made to avoid civil construction in this area. Third, it is likely that berm modifications will be required inside the Master Substation.

Region (c) is covered by a different methodology, which is underway. The longitudinal assessment shows deficiencies over the large beampipe upstream of M01 and over the M01 enclosure. If $3 \times 10^{13}$ intensity is used then the additional soil cover in this area is $1.5^{\prime}$. However if an intensity of $5 \times 10^{12}$ is used instead, then no remediation is required. Longitudinally, the enclosure M02 is adequate; however cross-sections and labyrinths are yet to be studied. Adoption of the methodology of E-Berm (electronic berm), which compares upstream and downstream intensities to determine if there are losses in any particular region, is seriously being considered from M03 to the Meson Detector Building. With E-berm in place this region will probably be shown to be adequately shielded 
throughout. It is noted that three E-Berms may be required, one for each of the primary Meson lines. At present only ME uses this methodology.

In region (c) for beam directed toward NM2, for which the $3 \times 10^{13}$ incident proton value must be used, preliminary results indicate the need for an additional one foot of soil cover over $60^{\prime}$ of NM1 and $1.5^{\prime}$ of cover over $100^{\prime}$ of NM2. As this beam undergoes no further splitting, it might be possible to use E-Berm here as well as in Meson.

\section{Controls}

The area of controls is one which has felt a major impact from the merging of the Accelerator and part of the Research Divisions. The control systems themselves, as well as the departments charged with maintaining and improving them, are in the process of being merged. The former Accelerator Division control system is known as ACNET; the RD system is called EPICURE. Of these two ACNET is in many ways the more sophisticated, and thus part of any beamline upgrade will involve migrating some EPICURE features onto ACNET and providing ACNET support to a wider area than formerly. A phased approach to retiring much of EPICURE in favor of ACNET has been begun and will be continuing in parallel with the other changes discussed in this report. The use of networked computers in both systems has made this merger realistic, a process which formerly would have been far more difficult and costly. The major steps consist of:

1. Providing a gateway allowing each control system to read data collected by the other. This functionality existed in the first half of 1996, and has been used increasingly throughout the 96-97 fixed target run.

2. Providing an ACNET front end with full read/write access to all EPICURE devices. Clearly, allowing write access is essential for any control, as opposed to data acquisition, system. This step is scheduled for completion in roughly September 1997.

3. Porting all generic EPICURE data handling to ACNET. After completion of this step there will be need for only one Save/Restore, Data Logger, Alarm System, etc. This work will be completed a few months after that on the EPICURE front end, presumably during the Main Injector shutdown.

4. Duplicating within ACNET the features of all important EPICURE application pages. On completion of this task EPICURE will be, at least from the perspective of an operator, gone (although its data acquisition hardware will remain). This project is a major one, with no completion date set at present. Presumably it will be partially finished when fixed target running returns after the shutdown.

As to controls of specific subsystems, the chief efforts will involve power supplies, BPMs and SWICs. Some power supplies will have ramps appropriate to Tevatron operation as is now taking place, some will ramp appropriately for $120 \mathrm{GeV}$ beam with a more rapid cycle rate, and a few may be required to do both. However, little new hardware will be required to complete this task and the insertion of new ramps does not require new software. Thus these changes will be relatively inexpensive and straightforward.

For the 96-97 run the SWICs of the former AD Switchyard have been outfitted with a new system of scanners. This system utilizes modern networking and processors, and is far more powerful than that which it replaced. It is intended to control the SWICs of the former $\mathrm{RD}$ with a similar system. 
The Switchyard BPMs have controls support which has fallen several years behind that of the accelerators. As part of a project to follow this one this system will be brought up to the present standard. The former EPICURE monitors will be integrated with the ACNET ones as part of the control system merger discussed above.

\section{Instrumentation}

It is not envisioned that instrumentation for the Switchyard and beamlines will have requirements differing significantly from those of the present. The challenges involve more rapid cycle time and in some cases higher instantaneous intensity for the Main Injector slow spill as compared with that of the Tevatron. In one regard the situation will be simpler than at present - there is no plan to send fast spill (appropriate for neutrino experiments) down the same lines as slow spill. It is presumed that the chief types of instrumentation required will be beam position/intensity monitors, SWICs and loss monitors.

Instrumentation will be required for the new $120 \mathrm{GeV}$ A0 bypass line through Transfer Hall and Enclosure B. Similarly, more detectors may be required in Meson lines than at present to accompany the new optics in that area. As a general rule SWICs will be more important for lines running $120 \mathrm{GeV}$ than they are now for $800 \mathrm{GeV}$, as understanding the increased widths of low energy beam profiles will be particularly important for tuning. It is felt that instruments from the decommissioned Neutrino line will be sufficient for the needs of the other areas, but a detailed design will be required to clearly establish this point and assure that nothing new needs to be constructed.

Typical Tevatron slow spill will last 40 seconds, while that from the Main Injector is specified as one second, with a possible increase to of order two seconds. Thus it will not be possible to sample the beam, especially with SWICs, so many times per cycle as with the Tevatron. There is no particular data collection limit, there being plenty of intensity, but there will be a processing problem. This can be addressed by building parallel subsystems, including enough processor power to handle the required throughput.

Current thinking on specific types of instrumentation follows:

BPMs: The tuning of BPMs should not be a major issue either for $120 \mathrm{GeV}$ beam or for regions through which both 120 and $800 \mathrm{GeV}$ beams pass. The $120 \mathrm{GeV} / 800 \mathrm{GeV}$ frequency difference is $1500 \mathrm{~Hz} v s$. a current system bandwidth of $400 \mathrm{kHz}$. The timing of these BPMs is delivered by the cable TV system and it is presumed that this will continue to be the case.

SEEDs: There are two SEEDs, presently in use in Neutrino, which will become available for new locations. Parts also exist to construct one more unit. The dimensions of the SEED ceramic boards are $4^{\prime \prime} \times 4^{\prime \prime}$ with an aperture of $11 / 16^{\prime \prime}$, as these devices were designed and built to display pretarget well focused beams (sigma less that $2-3 \mathrm{~mm}$ ). The wire spacings are $0.5,0.25$, and $0.125 \mathrm{~mm}$. For much larger beams the ceramic boards will have to be redesigned.

SWICs: There will be enough spare chambers to accommodate requests for the next run. Wire spacing for SWICs goes from $0.25 \mathrm{~mm}$ to $3 \mathrm{~mm}$ so it is possible to display much larger beams.

SEMs: There are presently in use many old detectors (some assembled in 1973) that should be replaced. If new SEMs are to be built. it would be advisable to redesign the unit to fit in a vacuum box. Presently the detectors require a beamline vacuum break and they are not moveable except for one detector in M-East. The current digitizer should also be modified to digitize incoming positive current. 
Two RF spill monitors will be employed, one each to provide spill intensity information to the Tevatron, as at present, and another to do the same for Main Injector. Others will be employed as required, such as to provide spill quality or frequency distribution information for particular beamlines.

\section{Acknowledgments}

The authors gratefully acknowledge the many hours of helpful discussions which have been essential to the production of this report. In particular our thanks go to Kevin Cahill,

Sam Childress, Mike Gerardi, Dave Johnson, George Krafczyk, Mike Martens, Larry Sauer and Jim Zagel. 


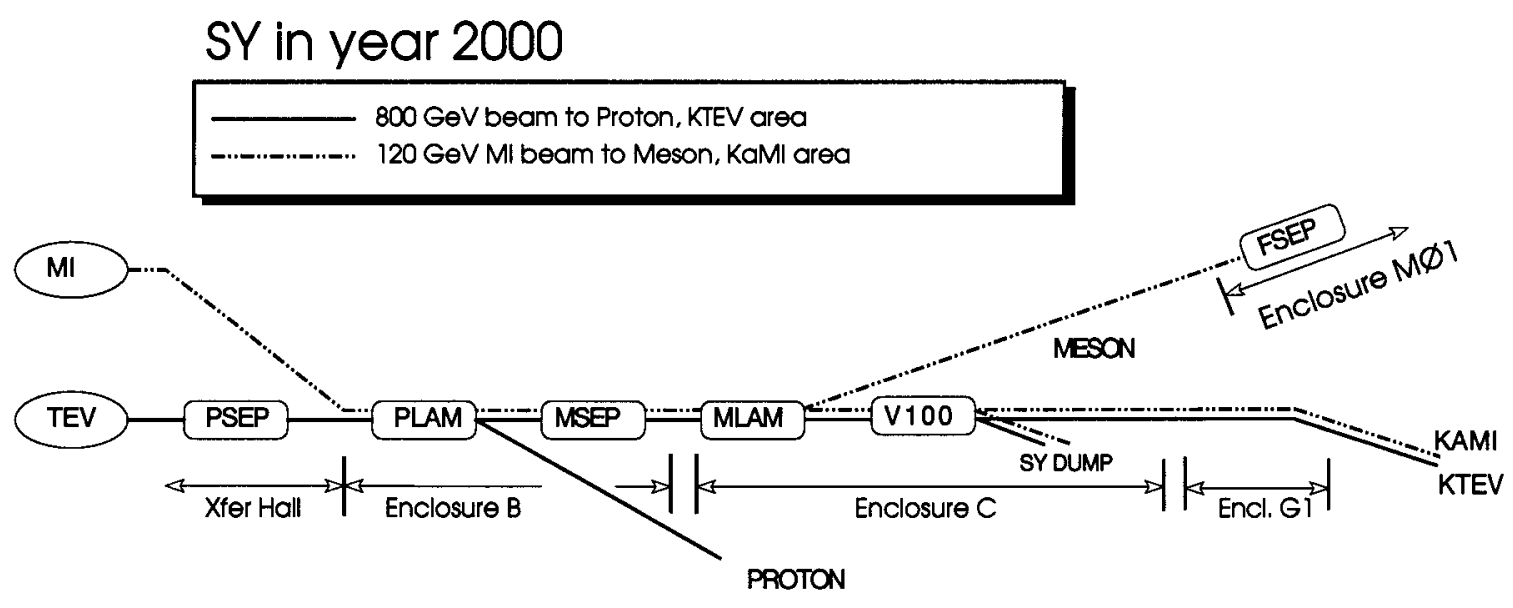

Figure 1. 


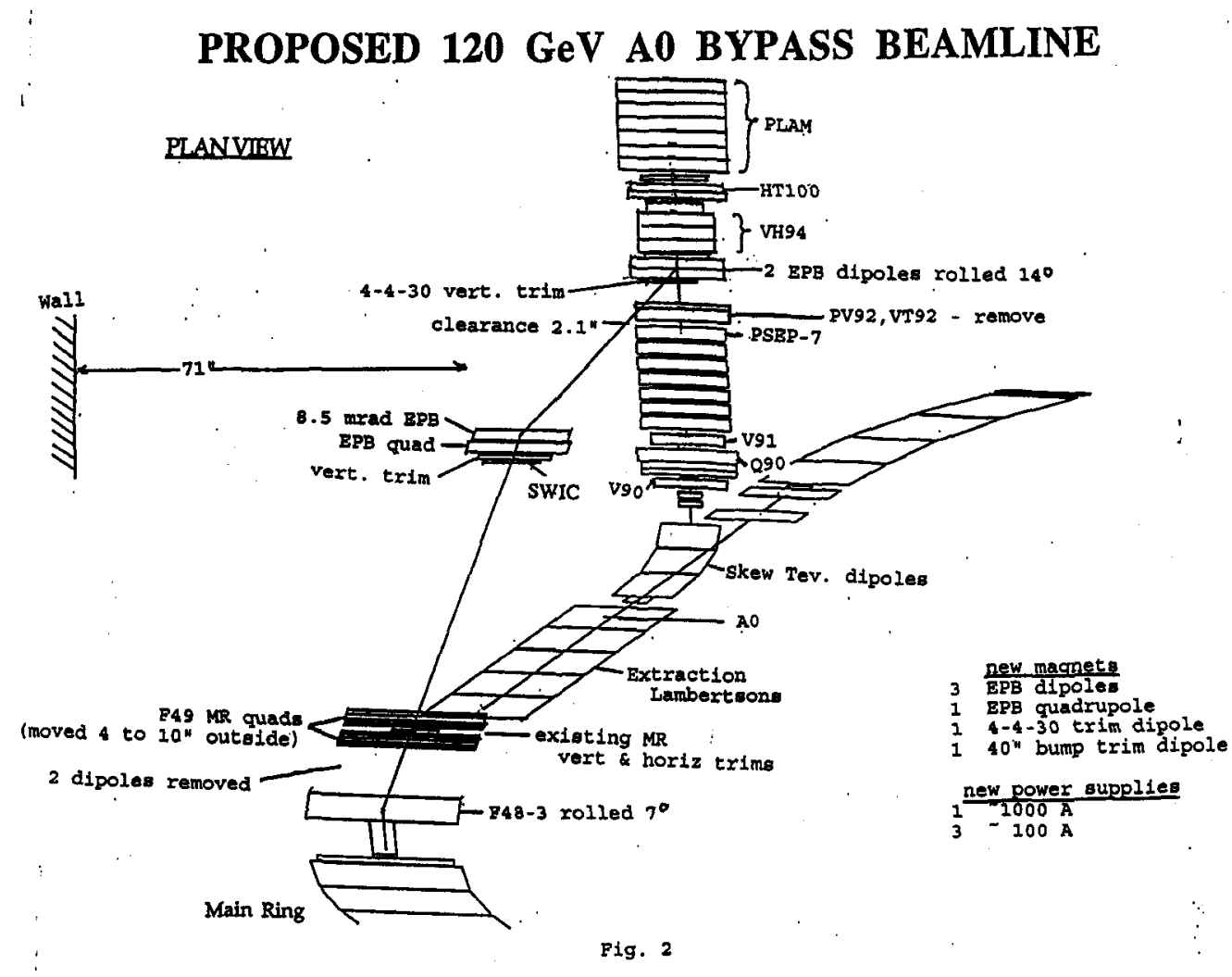

ALTERNATE 120 GeV AO BYPASS BEAMLINE

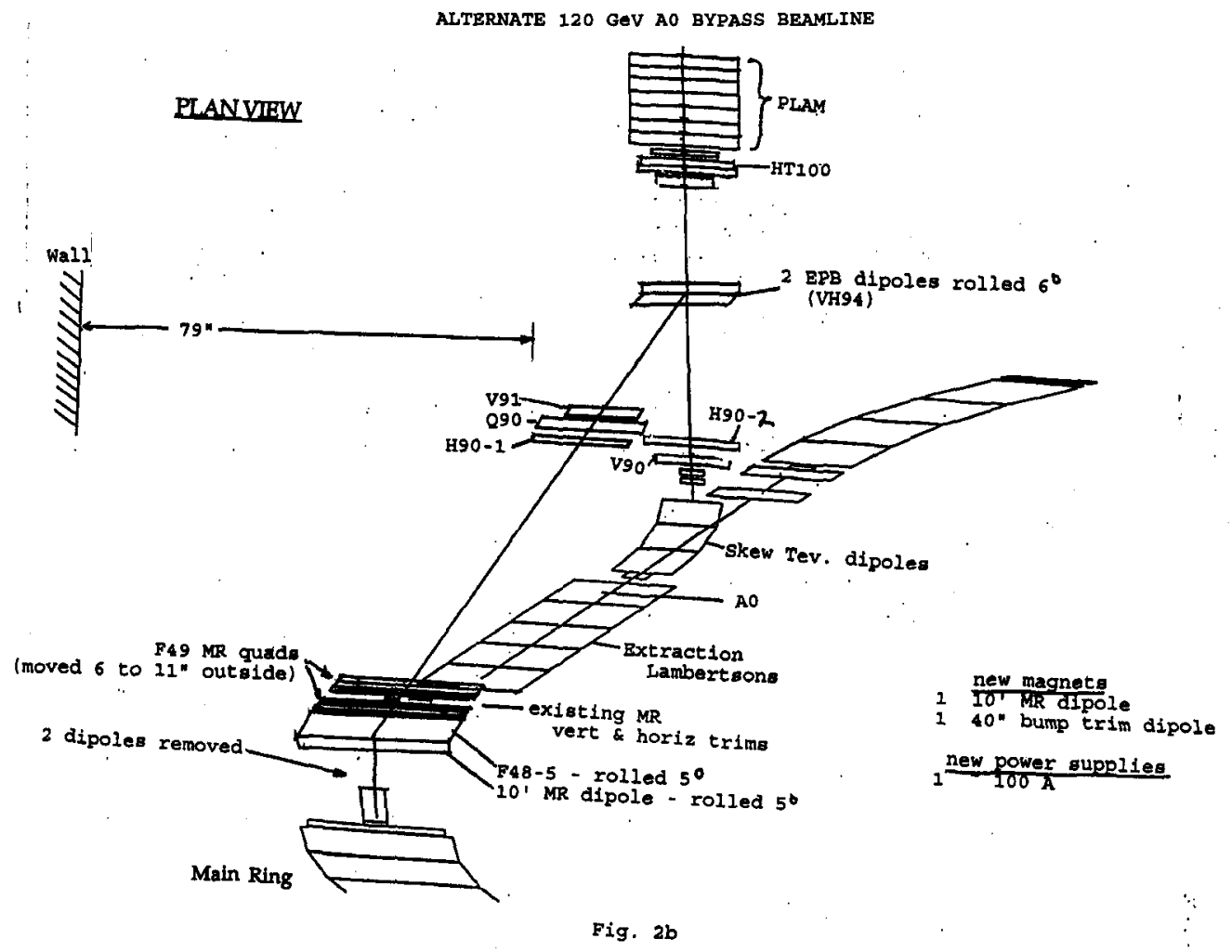


PROPOSED $120 \mathrm{GeV}$ AO BYPASS BEAMLINE

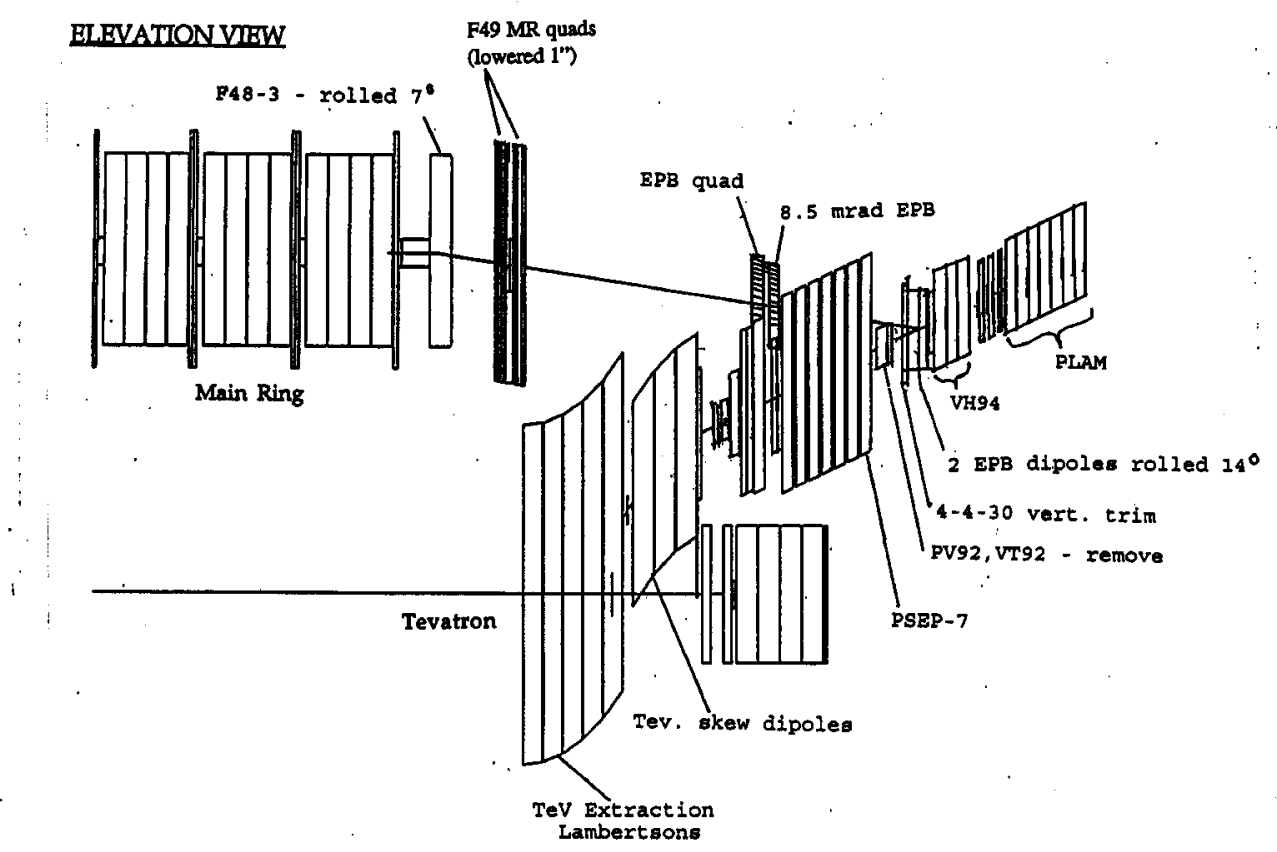

Fig. 3 


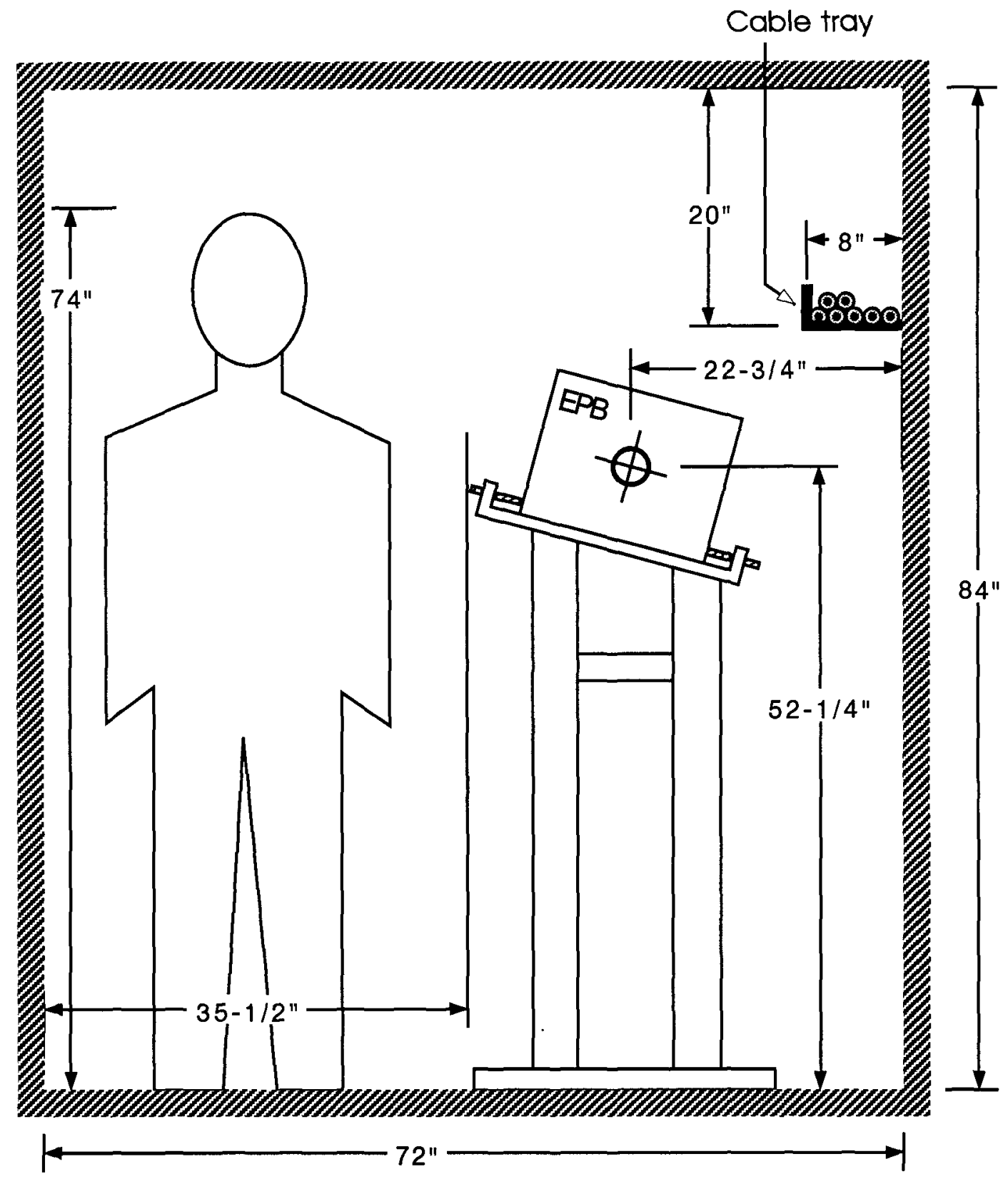

Figure 4. Typical cross-section indicating how rolled EPB dipoles fit in the tunnel. 
MR F11 to F17 section
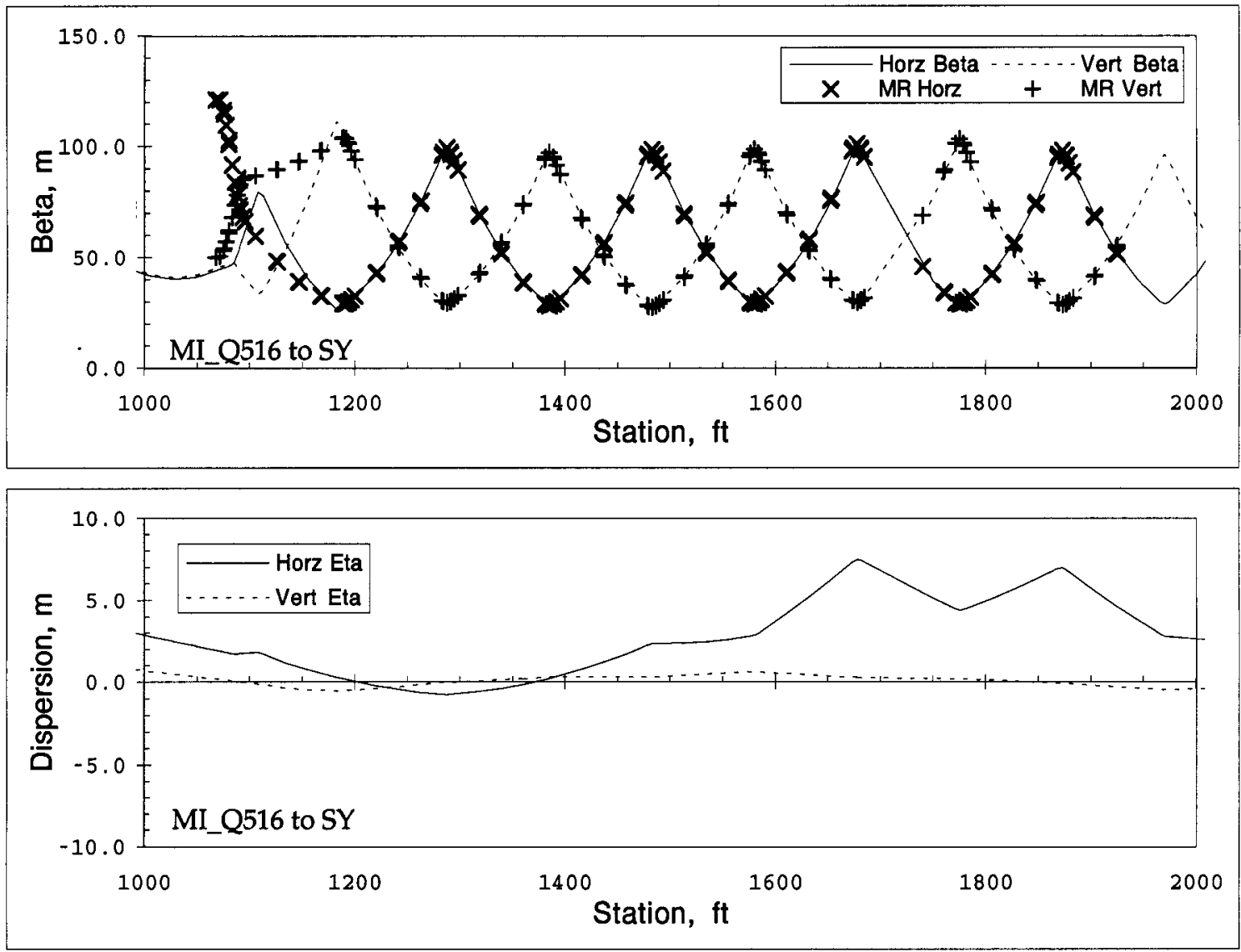

Figure 5. Optics of the MR remnant proceeding downstream from F11 
Fit at $F 48, F 49 A, F 49 B, F 49 C, \& F 49 D$
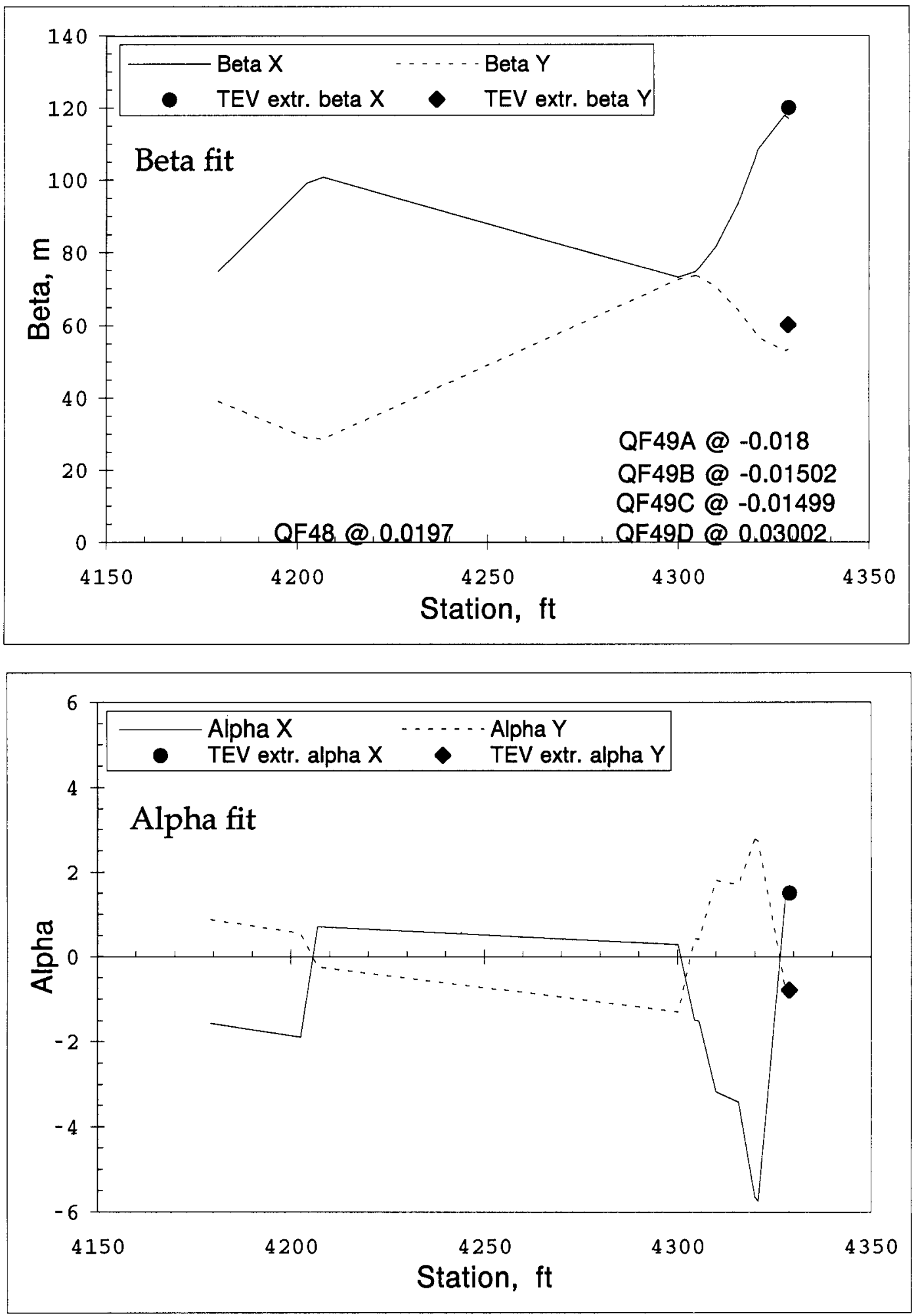

Figure 6. A demonstration of the extent to which MR optics agrees with that of the TeV near A0 


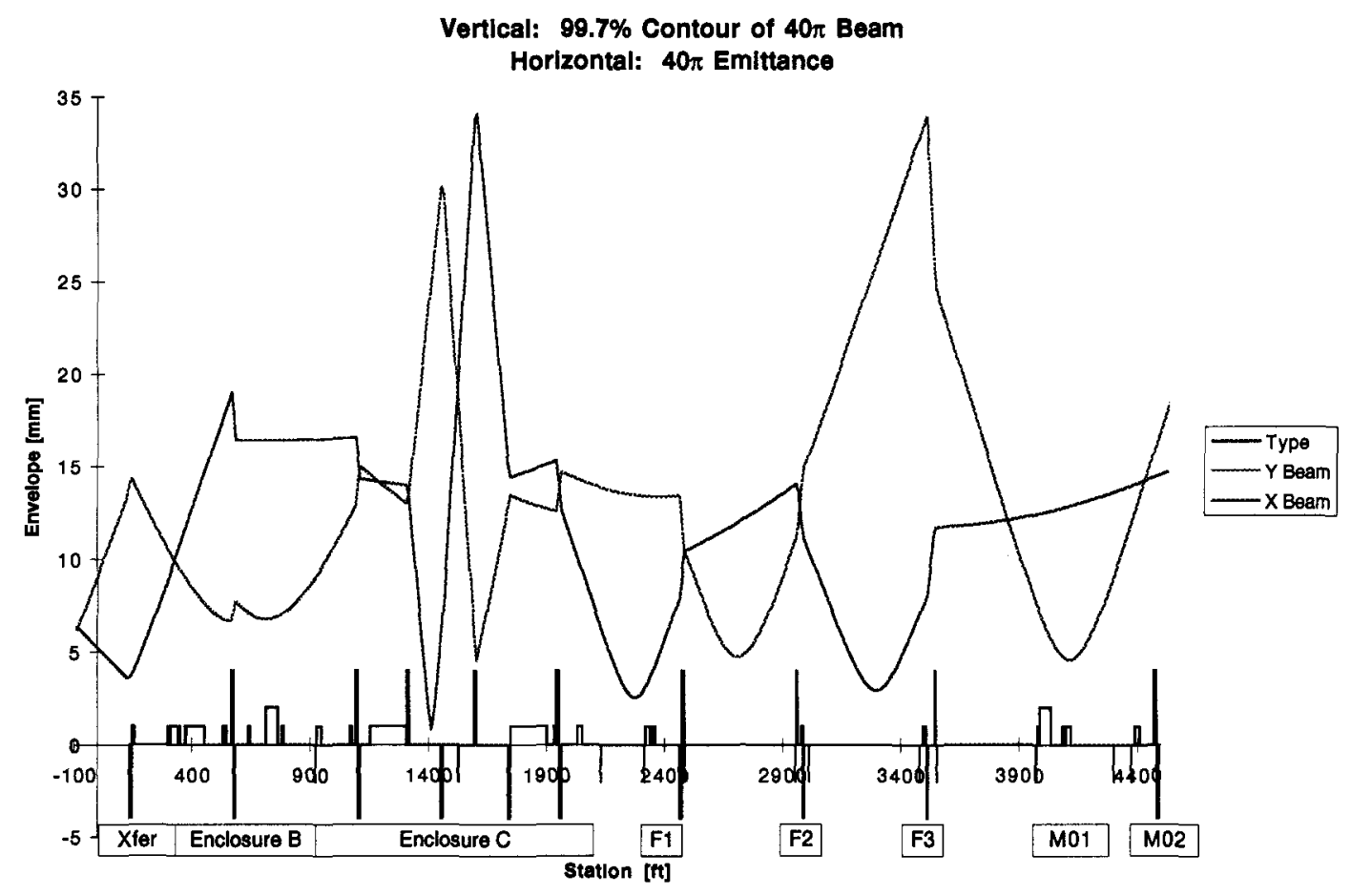

Figure 7. Meson line optics beginning at a point near AO

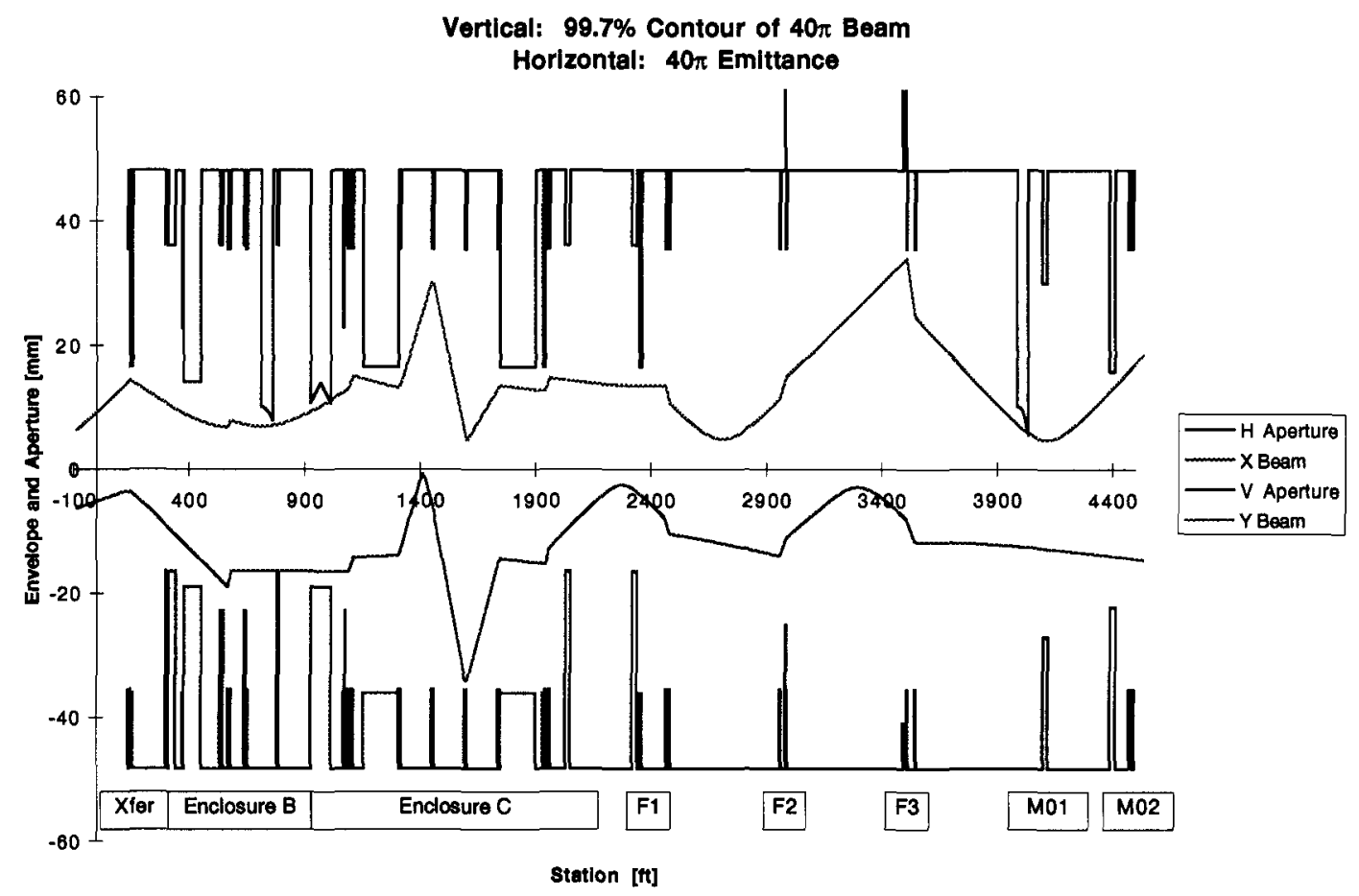

Figure 8. Comparison of Meson line envelopes with critical apertures 
Vertical: $99.7 \%$ Contour of $40 \pi$ Beam

Horlzontal: $40 \pi$ Emittance

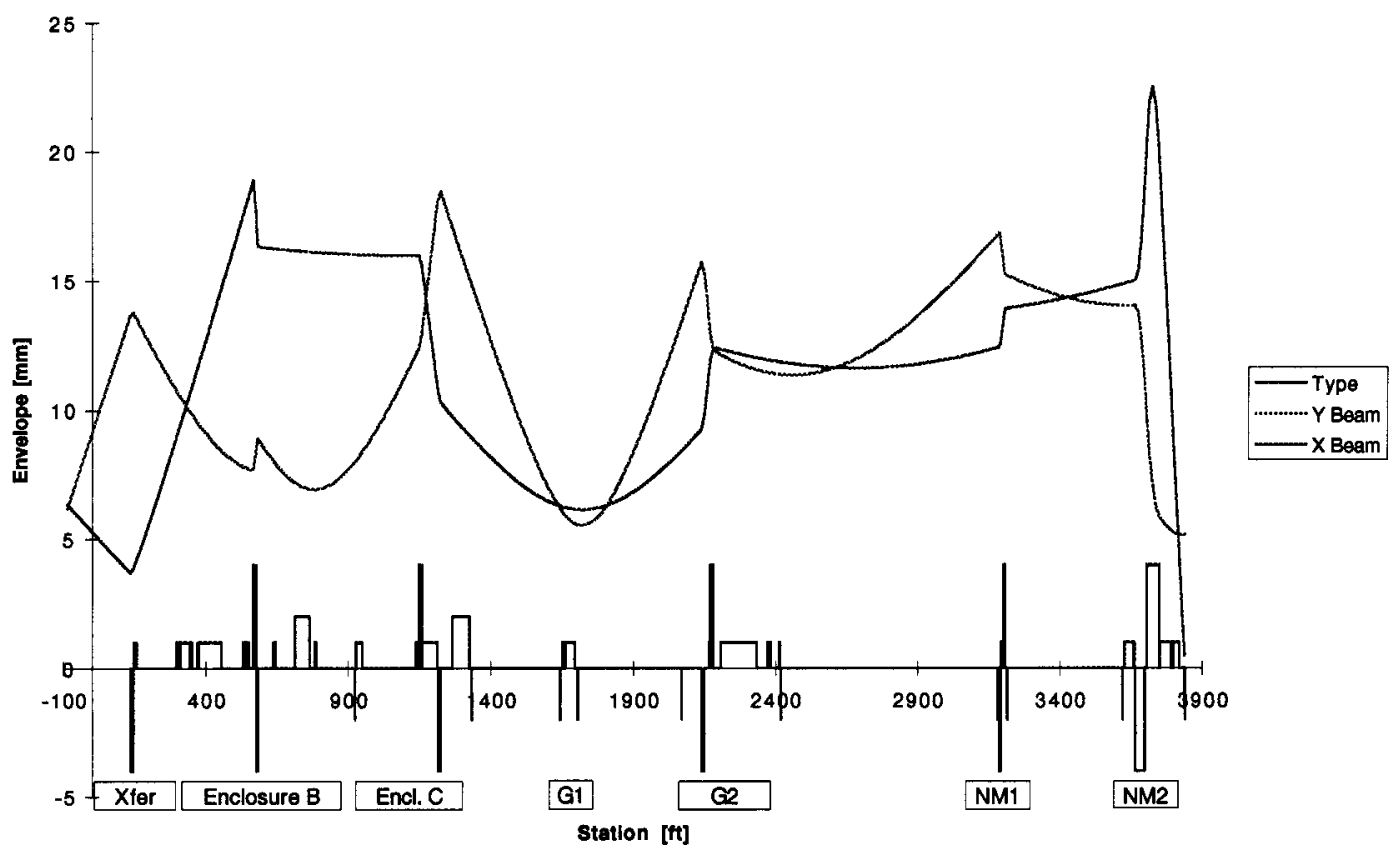

Figure 9. Muon line optics beginning at a point near $A 0$

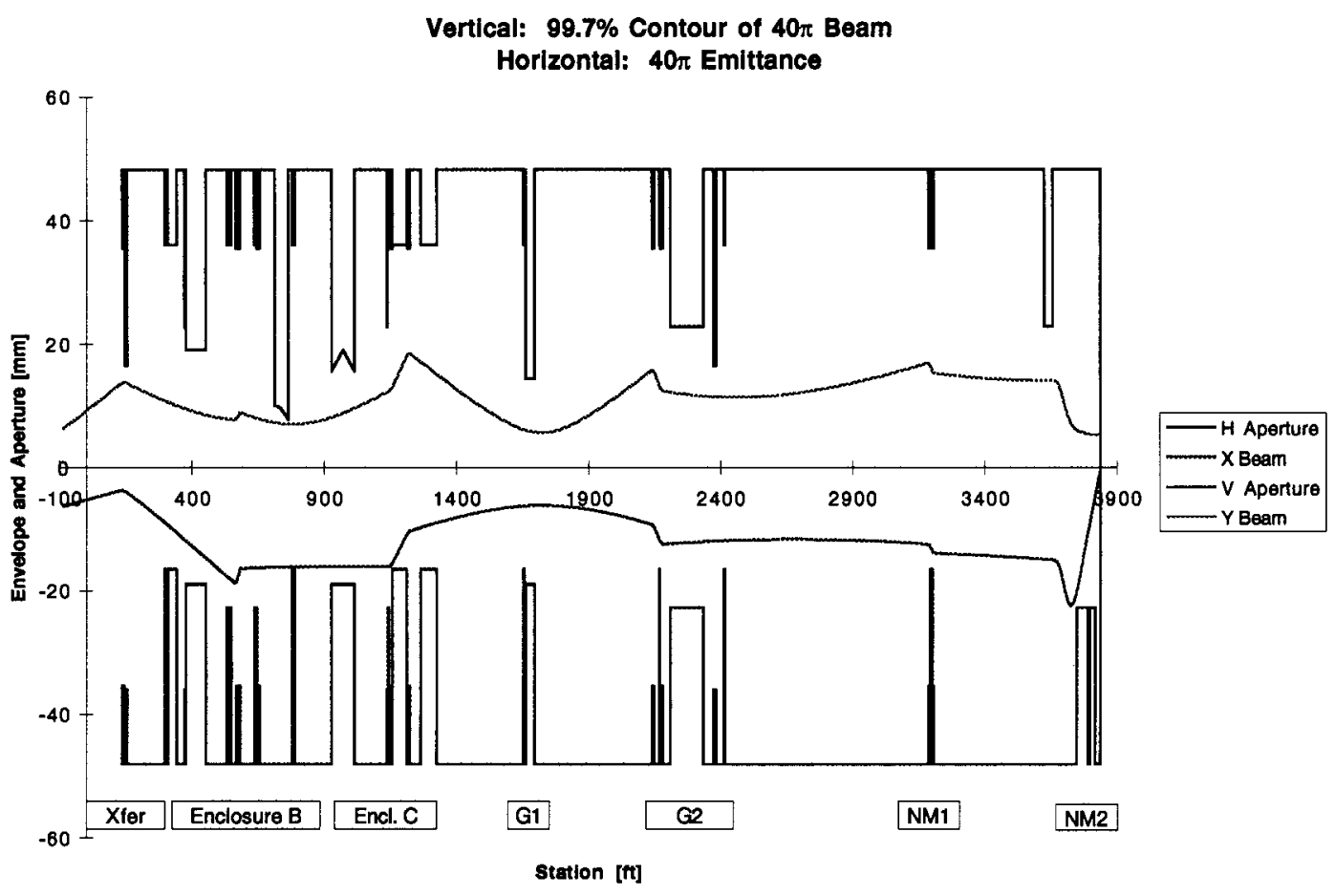

Figure 10. Comparison of Muon line envelopes with critical apertures 


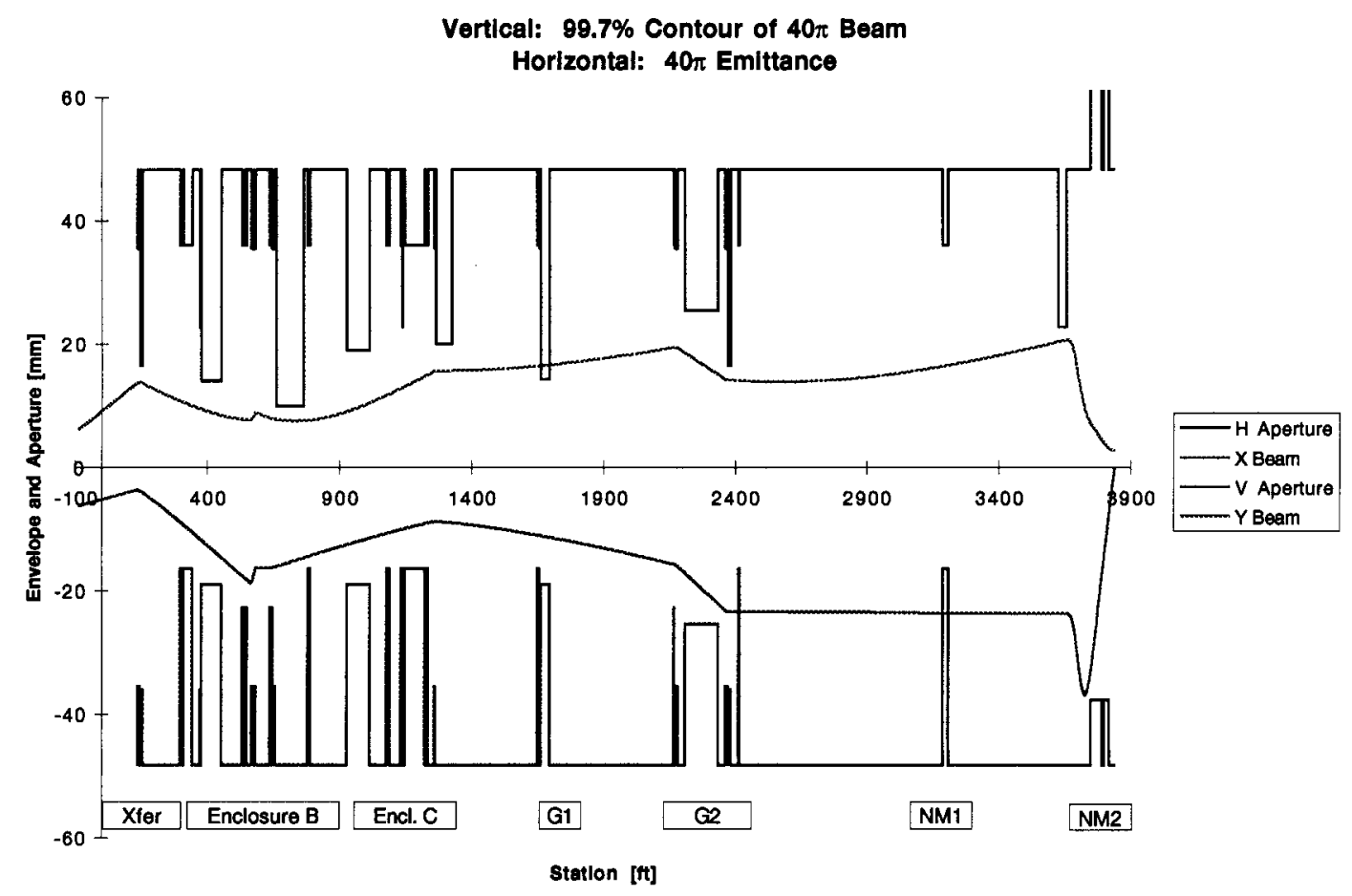

Figure 11. Comparison of Muon line envelopes with critical apertures using only the quadrupoles which exist today 